\title{
PREPARATION OF FLUORESCENT POLYSTYRENE MICROSPHERES BY IMPROVING SWELLING PERFORMANCE BASED ON MOLECULAR WEIGHT AND SURFACE CARBOXYL GROUP CONTENT
}

Fei Suna, Chao Feng ${ }^{\mathrm{b}}$, Yang Wanga ${ }^{\mathrm{a}}$, Xue Yang ${ }^{\mathrm{a}}$ and Hong Zhao ${ }^{\mathrm{a}, *, \mathbb{1}}$

${ }^{a}$ School of Chemistry and Chemical Engineering, Southeast University, Nanjing 211189, China

${ }^{\mathrm{b}} \mathrm{S}$ chool of Materials and Chemical Engineering, Bengbu University, Bengbu 233030, PR China

Recebido em 07/05/2021; aceito em 16/07/2021; publicado na web em 13/08/2021

\begin{abstract}
The fluorescent microspheres are prepared by the swelling-evaporation method. The optimal swelling conditions are obtained by researching related factors, such as types and dosages of swelling agent, dosages of fluorescent substance, swelling time and swelling temperature. Considering that the structure of microspheres can influence the swelling properties, the paper mainly focuses on the effect of molecular weight and surface carboxyl group content of polystyrene microspheres on swelling properties. When the molecular weight of microspheres is $75276 \mathrm{~g} \mathrm{~mol}^{-1}$, the density of carboxyl group on their surface is $6.94 \times 10^{-4} \mathrm{~mol} \mathrm{~g}^{-1}$, and the doping amount of europium complex reaches the maximum of $24.9 \mathrm{mg} \mathrm{g}^{-1}$ with the strongest fluorescence intensity. Meanwhile, the as-prepared fluorescent microspheres have high monodispersity, uniform particles size and high thermal stability (the decomposition temperature is $385^{\circ} \mathrm{C}$ ), which sets up the good foundation for biomolecule detection. So, this work provides a significant experience for the preparation of fluorescent microspheres.
\end{abstract}

Keywords: swelling method; fluorescence intensity; optimal conditions; doping of europium complex.

\section{INTRODUCTION}

In recent years, fluorescent microspheres have gained increasing attention for their wide applications in the biomolecules detection, imaging, fluid mechanics studies and standardization of fluorescentbased instruments. ${ }^{1-6}$ The choice of fluorescent material and polymer matrix is important to prepare fluorescent microspheres with strong fluorescence intensity and high stability. Compared with other traditional fluorescent materials, the luminescent rare earth complexes ${ }^{7}$ are widely used for their unique spectral properties, such as extremely narrow and intense emission bands, relatively long lifetimes and large stokes shift. Among many different polymers, polystyrene which is rigid, ${ }^{8-10}$ transparent, and easy to functionalize ${ }^{11}$ can provide a stable environment to protect the pure rare earth organic complexes. The researches indicate that their photoluminescence properties and thermal stability are improved when rare earth complexes are incorporated into the polystyrene microspheres. ${ }^{12-17}$

To date, several different fabrication methods for preparation fluorescent microspheres have been developed, such as encapsulation of europium complexes in the monomer before polymerization, ${ }^{18-21}$ copolymerization with reactive fluorophores, ${ }^{22,23}$ and swelling preexisting particles. ${ }^{24-27}$ The swelling-evaporation method is a simple, versatile and effective technique. However, the swelling process is easily affected by conditions mentioned above and the molecular weight of microspheres. Considering the application of fluorescent microspheres in biomedicine, it is necessary to modify the surface of the microspheres with functional groups. ${ }^{28-30}$ There are few researches on the effect of functional groups on the surface of microspheres on their swelling performance. The influence of functional group content on the surface of microspheres on the swelling properties is also the focus of our research in the paper.

Herein, in order to research the effect of molecular weight and surface carboxyl group content of the microspheres on the swelling properties, series of microspheres with different surface carboxyl contents and molecular weight were prepared in this paper. The

*e-mail: zhaohong@seu.edu.cn luminescent composite microspheres of Eu(NTFA) $)_{3}$ Phen are successfully prepared according to the optimal swelling conditions. And characterization of the properties of microspheres revealed that when the molecular weight of the microspheres is $75276 \mathrm{~g} \mathrm{~mol}^{-1}$, the surface carboxyl group content is $6.94 \times 10^{-4} \mathrm{mmol} \mathrm{mg}^{-1}$, the fluorescent microspheres have excellent fluorescence properties, high stability and abundant functional groups, which may extend the applications of fluorescent microspheres in biomolecules detection.

\section{EXPERIMENTAL}

\section{Materials}

10-phenanthroline (Phen), $\mathrm{Eu}_{2} \mathrm{O}_{3}(99.9 \%)$ and 4,4,4-trifluoro1-(2-naphthyl)-1,3-butanedione (NTFA) are purchased from Aladdin chemical Reagent Company. Styrene is purified with alkaline alumina by column chromatography to remove inhibitor and stored at $-5^{\circ} \mathrm{C}$. Methacrylic acid (MAA) is purified by passing it through an inhibitor removal column and stored at $-5{ }^{\circ} \mathrm{C}$. All other commercially available reagents are of reagent grade and are used without further purification.

\section{Preparation of europium organic complex}

The alcoholic solution of $\mathrm{EuCl}_{3}$ was prepared by dissolving $\mathrm{Eu}_{2} \mathrm{O}_{3}(0.5 \mathrm{mmol}, 176.0 \mathrm{mg})$ in concentrated hydrochloric acid $(\mathrm{HCl}$, $10 \mathrm{~mL}$ ), heating to remove the acid and adding to appropriate ethanol (20 mL). Eu(NTFA) $)_{3}$ Phen (NTFA = 4,4,4-trifluoro-1-(2-naphthyl)1,3-butanedione, Phen $=1,10$-phenanthroline) was synthesized and characterized according to the published methods. ${ }^{31}$ The NTFA ( $3 \mathrm{mmol}, 798.7 \mathrm{mg}$ ) and Phen $(1 \mathrm{mmol}, 198.2 \mathrm{mg}$ ) were dissolved in absolute ethanol and then heated to $70{ }^{\circ} \mathrm{C}$. The $\mathrm{pH}$ value was adjusted to $6.0-7.0$, using $1.0 \mathrm{~mol} \mathrm{~L}^{-1} \mathrm{NaOH}$ aqueous and then the $\mathrm{EuCl}_{3}(1 \mathrm{mmol}, 258.5 \mathrm{mg})$, aqueous was added dropwise. The reaction was performed $6 \mathrm{~h}$ at $70^{\circ} \mathrm{C}$. After the mixture cooled down to room temperature, the precipitate was filtered under reduce pressure and washed 3 times with $50 \mathrm{~mL}$ ethanol and $50 \mathrm{~mL}$ water respectively to remove unreacted raw materials, and then dried in a vacuum oven. 
Preparation of monodisperse poly (styrene-co-methylacrylic acid) (PSM) microspheres with different surface carboxyl group contents

The monodisperse PSM microspheres with different surface carboxyl group contents are prepared by soap-free emulsion polymerization $^{32}$ to study the effect of the surface carboxyl group content on the swelling property. The polymerization reaction is carried out in a $100 \mathrm{~mL}$ three-necked flask equipped with a nitrogen inlet and a reflux condenser. $2 \mathrm{~mL}$ of styrene, $25 \mathrm{~mL}$ deionized water and $25 \mathrm{~mL}$ ethanol are poured into the flask, purging nitrogen for 10 min to remove the oxygen in the flask. The flask is placed in an oil bath and until the temperature reaches $80^{\circ} \mathrm{C}, 0.5 \mathrm{~mL}$ aqueous solution containing $40 \mathrm{mg}$ of APS is charged. After $2.5 \mathrm{~h}, 0.1 \mathrm{~mL}$ of MAA is added into the flask, and the polymerization is maintained under nitrogen atmosphere at a stirring rate of $200 \mathrm{rpm}$ for $10 \mathrm{~h}$. The formed microspheres are washed 3 times with $45 \mathrm{~mL}$ ethanol and $45 \mathrm{~mL}$ deionized water respectively, then collected by centrifugation and stored at $-5{ }^{\circ} \mathrm{C}$. The monodisperse PSM microspheres with different surface carboxyl group contents are prepared similarly, by changing the volume of methacrylic acid (MAA).

\section{Determination of surface carboxyl group content in PSM microsphere emulsion}

The conductometric titration is used to determine the content of carboxyl group on the surface of microspheres. The specific experimental steps are performed according to the method reported in the reference. ${ }^{33}$ First, weigh $144 \mathrm{mg}$ of sample microspheres in a $100 \mathrm{~mL}$ beaker, and dilute to $40 \mathrm{~mL}$ with deionized water. $5 \mathrm{~mL} 0.1 \mathrm{~mol} \mathrm{~L}^{-1}$ sodium hydroxide solution is added in the diluted microspheres emulsion and mechanically stir for $2 \mathrm{~h}$. Then, the obtained emulsion is titrated with $0.1 \mathrm{~mol} \mathrm{~L}^{-1} \mathrm{HCl}$ solution. The reaction of sodium carboxylate and hydrochloric acid occurs in the stage of constant conductivity, and the volume of hydrochloric acid consumes $\mathrm{V}$.

The formula for calculating the surface carboxyl group content is as follows:

Surface carboxyl content $=(\mathrm{V} \times \mathrm{C}) / \mathrm{M}$

where $\mathrm{V}$ is the volume of $\mathrm{HCl}$ titrant in latex requires to neutralize the surface bound carboxyl, $\mathrm{C}$ is the concentration of the $\mathrm{HCl}$ titrant, $\mathrm{M}$ is the weight of sample microspheres.

\section{Preparation of monodisperse poly (styrene-co-methylacrylic acid) microspheres with different molecular weight}

In order to study the influence of molecular weight of microspheres on the swelling property, the monodisperse PSM microspheres with different molecular weight are prepared in alcohol/water medium with ammonium persulfate (APS) as initiator. $2 \mathrm{~mL}$ styrene, $25 \mathrm{~mL}$ deionized water and $25 \mathrm{~mL}$ ethanol are poured into flask. As the temperature is heated to $80{ }^{\circ} \mathrm{C}$ under nitrogen atmosphere, $0.5 \mathrm{~mL}$ aqueous solution containing 20 $\mathrm{mg}$ of APS is charged. After $2.5 \mathrm{~h}, 0.4 \mathrm{~mL}$ of MAA is added, and the polymerization is kept for $10 \mathrm{~h}$ at $80{ }^{\circ} \mathrm{C}$ under nitrogen atmosphere. The formed microspheres are washed 3 times with $45 \mathrm{~mL}$ ethanol and $45 \mathrm{~mL}$ deionized water repetitively, and then collected by centrifugation and stored at $-5{ }^{\circ} \mathrm{C}$. The monodisperse PSM microspheres with different molecular weight are prepared similarly by changing the concentration of initiator. ${ }^{34}$

\section{Preparation of monodisperse surface carboxylated fluorescent} microspheres (Eu/PS-COOH)

Figure 1 shows the schematic illustration of the preparation of fluorescent microspheres. Fluorescent microspheres are prepared by swelling-evaporation approach. The carboxyl group-functionalized microspheres $(100 \mathrm{mg})$ are dispersed in swelling medium $(10 \mathrm{~mL}$ water), follow by the addition of dichloromethane solution $(1 \mathrm{~mL})$ containing of europium complex $(10 \mathrm{mg})$. The mixed solution is sealed in a flack and stirred at $40{ }^{\circ} \mathrm{C}$ for $2 \mathrm{~h}$ to swell microspheres, then remove the dichloromethane by reducing pressure distillation. The formed microspheres are firstly washed with $60 \mathrm{~mL}$ alcohol for four times to remove the residual europium complex, then continue to wash with $30 \mathrm{~mL}$ deionized water and stored at $-5{ }^{\circ} \mathrm{C}$ for later characterization.

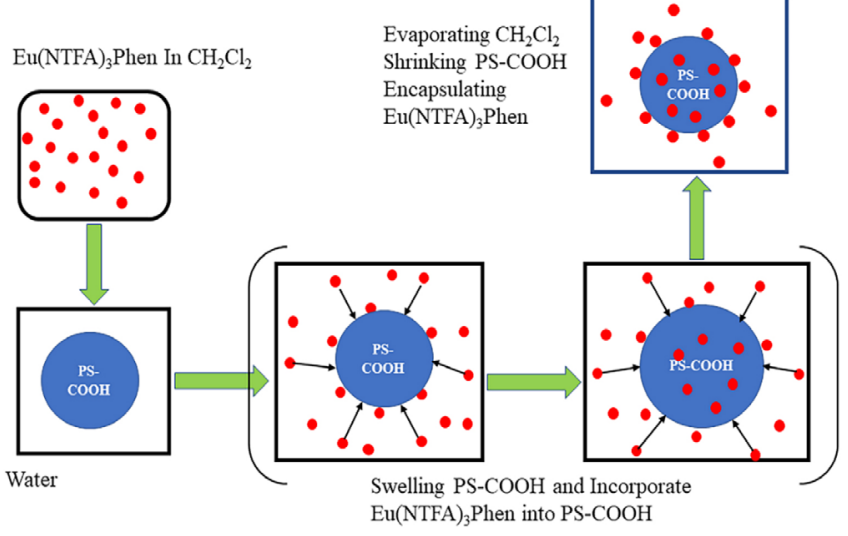

Figure 1. Schematic illustration of preparation of fluorescent microsphere by the swelling-evaporation method

\section{Characterization}

Field emission scanning electron microscope (FESEM, Inspect F50, FEI) is applied to observe the morphologies of polymer microspheres. The internal structure of polymer microspheres is measured by high-resolution transmission electron microscope (HRTEM, G2-20, FEI). The fluorescence excitation and emission spectra are collected through the fluorescence spectrophotometer (Fluoromax-4, Horiba). The average molecular weight of polymer microspheres is measured by gel permeation chromatography (GPC, PL-GPC220, Polymer Laboratories). Having a differential refractometer as a detector, tetrahydrofuran is used as eluent at a flow rate of $1.0 \mathrm{~mL} \mathrm{~min}{ }^{-1}$. The thermal stability of fluorescent microspheres is investigated by a thermogravimetric analyzer under $\mathrm{N}_{2}$ atmosphere, between the temperature of 50 and $800{ }^{\circ} \mathrm{C}$, with a heating rate of $10{ }^{\circ} \mathrm{C} \mathrm{min}^{-1}$.

\section{RESULTS AND DISCUSSION}

\section{Fluorescence properties of europium complex}

The fluorescence excitation spectra and emission spectra of Eu(NTFA) ${ }_{3}$ Phen in DMF has been measured. As shown in Figure 1Sa, the maximum excitation wavelength of $\mathrm{Eu}(\mathrm{NTFA})_{3}$ Phen is $340 \mathrm{~nm}$. Upon excitation at $340 \mathrm{~nm}$, europium complex of Eu(NTFA) $)_{3}$ Phen exhibited the emission spectra in Figure $1 \mathrm{Sb}$, and there were five absorption peaks at $579 \mathrm{~nm}, 593 \mathrm{~nm}, 614 \mathrm{~nm}, 651 \mathrm{~nm}, 701 \mathrm{~nm}$, which corresponds to the five transitions of $5 \mathrm{D}^{\circ} \rightarrow 7 \mathrm{~F}^{\circ}, 5 \mathrm{D}^{\circ} \rightarrow 7 \mathrm{~F}^{1}$, $5 \mathrm{D}^{\circ} \rightarrow 7 \mathrm{~F}^{2}, 5 \mathrm{D}^{\circ} \rightarrow 7 \mathrm{~F}^{4}$ and $5 \mathrm{D}^{\circ} \rightarrow 7 \mathrm{~F}^{5}$ of $\mathrm{Eu}^{3+}$, respectively. Among them, $5 \mathrm{D}^{\circ} \rightarrow 7 \mathrm{~F}^{2}$ belongs to the electric dipole transition with the peak at $614 \mathrm{~nm}$, and its emission intensity is the strongest. To better 

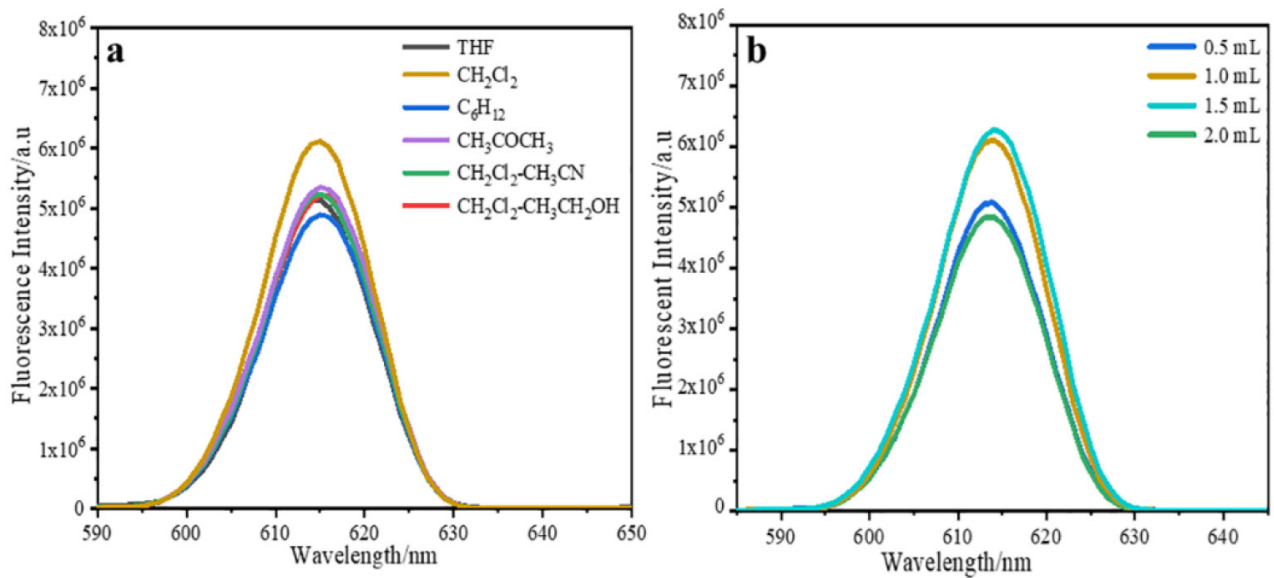

Figure 2. (a) Fluorescence emission spectra of fluorescent microspheres swelled by different swelling agent; (b) Fluorescence emission spectra of fluorescent microspheres swelled by different volumes of dichloromethane

evaluate the fluorescence intensity of microspheres, herein we just considered the strongest fluorescence intensity of peak at $614 \mathrm{~nm}$. In the experiment, we found that the doping of europium complex inside the microsphere does not affect its fluorescence behavior, and the emission band of the as-prepared fluorescence microsphere is still at $614 \mathrm{~nm}$ upon excitation at $340 \mathrm{~nm}$. In addition, according to the comment, we measure the fluorescence spectrum of the blank microspheres. As shown in the Figure $2 \mathrm{~S}$, there is no fluorescence between $580 \mathrm{~nm}$ to $650 \mathrm{~nm}$ on the polystyrene microspheres when excited at $340 \mathrm{~nm}$.

\section{Optimization of the microspheres swelling conditions through the single factor method}

Before researching the effect of molecular weight and surface carboxyl content of microspheres on the swelling properties, the external factors that can affect the swelling performance and fluorescent property are first studied through the single factor method to optimize the swelling conditions, for the swelling process is easily affected by external factors mentioned above. The microspheres used in the following researches are all $0.1 \mathrm{~g}$, which surface carboxyl group content is $6.94 \times 10^{-4} \mathrm{~mol} \mathrm{~g}^{-1}$ and molecular weight is $71219 \mathrm{~g} \mathrm{~mol}^{-1}$. The concentration of fluorescent microspheres used for fluorescence testing is $3.3 \times 10^{-10} \mathrm{~mol} \mathrm{~L}^{-1}$.

In this paper, dichloromethane, tetrahydrofuran (THF), acetonitrile, 1,2-dichloroethane, acetone, ethanol, and cyclohexane are initially selected as swelling agents. The fluorescent microspheres are successfully prepared with these solvents and characterized by fluorescence spectrophotometer. By comparing these results (Figure 2a), the fluorescent microspheres are prepared with dichloromethane solution of europium complex with the strongest fluorescence intensity. Therefore, dichloromethane is chosen as the swelling agent in the following experiments. Afterward, the addition amount of dichloromethane is $0.5 \mathrm{~mL}, 1.0 \mathrm{~mL}, 1.5 \mathrm{~mL}$, and $2 \mathrm{~mL}$ to research the effect of swelling agent content on the swelling properties of microspheres. The fluorescent microspheres are prepared by swelling the polymer matrix $(0.1 \mathrm{~g})$ with different volumes of dichloromethane and characterized by TEM and fluorescence spectrophotometer. As shown in Figure 2b, when the volume of dichloromethane is $1.5 \mathrm{~mL}$, the fluorescence intensity of microspheres is the strongest. When the volumes of dichloromethane are $0.5 \mathrm{~mL}$, $1.0 \mathrm{~mL}$ and $1.5 \mathrm{~mL}$, the resulting microspheres show uniform diameter and regular morphology (Figure 3a, 3b, 3c). However, when the volume of dichloromethane is $2 \mathrm{~mL}$, the obvious conglutination of the fluorescent microspheres appears (Figure 3d). Therefore, the volume of the dichloromethane main affects the morphology of the microspheres and the volume of dichloromethane used in the following experiments is $1.5 \mathrm{~mL}$.

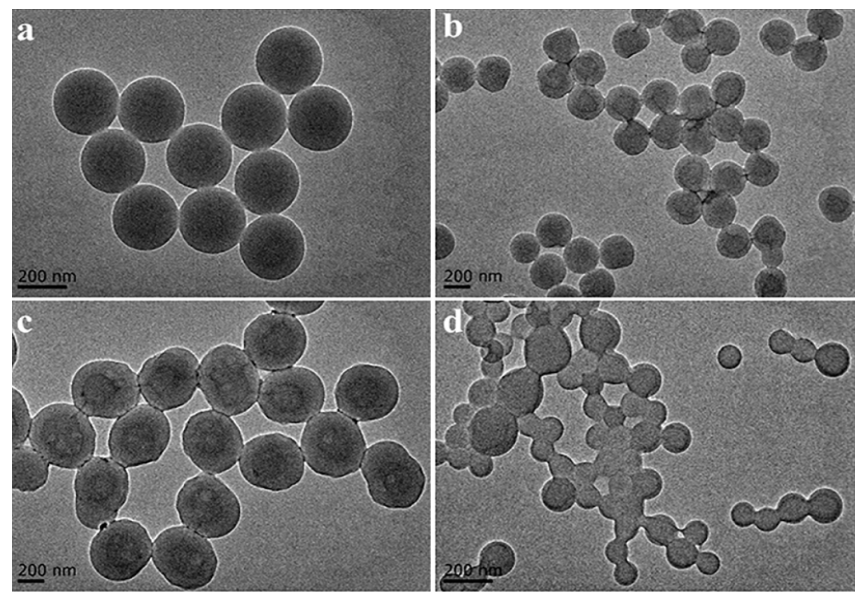

Figure 3. TEM images of fluorescent microspheres swelled by different volumes of dichloromethane solution ( $a: 0.5 \mathrm{~mL}, b: 1.0 \mathrm{~mL}, c: 1.5 \mathrm{~mL}$, and d: $2.0 \mathrm{~mL}$ )

The mass of europium complex has a great impact on the fluorescence intensity of the microspheres. The fluorescent microspheres are prepared with different dosages of europium complex and characterized by fluorescence spectrophotometer. As shown in Figure 4a, when the amount of europium complex is less than $15 \mathrm{mg}$, the fluorescence intensity of the microspheres is increase gradually and reaches the maximum when $15 \mathrm{mg}$ of europium complex is added, and the fluorescence intensity of the microspheres do not increase with the augment of the amount of europium complex. The result indicates that the doping amount has reached the critical saturation value. If the amount of europium complex is further increased, the fluorescence intensity of the microspheres became decreasing, it may be that the aggregation of europium complex caused fluorescence quenching. ${ }^{35,36}$ As we know, when the concentration of dye is too high, the concentration quenching will occur, in which the luminescence will be quenched by the energy migration among the molecules. Therefore, when europium complex molecules aggregate, fluorescence quenching will be caused by energy transfer. Therefore, we believe that the optimal doping amount of europium complex is $15 \mathrm{mg}$.

The swelling temperature has little impact on the fluorescence intensity of microspheres and the fluorescence intensity of the microspheres reaches the maximum value when the swelling 
temperature is $50^{\circ} \mathrm{C}$ (Figure $4 \mathrm{~b}$ ). This because when the temperature is lower, the swelling speed of europium complex will be affected, the diffusion speed of the europium complex will be increased with the increase of temperature, and is advantageous to the $\mathrm{Eu}(\mathrm{NTFA})_{3}$ phen molecules diffusion into the microspheres. However, the binding strength between europium ion and ligand became weakened under higher temperature, which resulted in an increase in the distance between europium ion and ligand. Thus, reducing the antenna effect of the europium complex, and thus reducing the process of the ligand receiving energy to excite europium ions, as a result, its fluorescence intensity is gradually weakened. Therefore, the optimal swelling temperature is $50{ }^{\circ} \mathrm{C}$. The swelling time is $3 \mathrm{~h}$ and the fluorescence intensity of the microspheres after extending swelling time is almost unchanged (Figure 4c). At this time, the doping amount has reached the critical saturation value and the swelling process is finished. In summary, the optimal swelling conditions of $0.1 \mathrm{~g}$ microspheres matrix are obtained in the research: $1.5 \mathrm{~mL}$ of dichloromethane as the swelling agent, the dosage of europium complex is $15 \mathrm{mg}$ and swell for $3 \mathrm{~h}$ at $50^{\circ} \mathrm{C}$.

\section{Effect of the surface carboxyl group content of microspheres on the swelling properties of microspheres}

In order to obtain microspheres with different surface carboxyl group contents, the polystyrene microspheres with different surface carboxyl group contents are prepared by soap-free emulsion polymerization via changing the volume of MAA. The surface carboxyl group content of PSM microspheres determined by the conductometric titration are summarized in Figure 5a. The surface carboxyl content of microspheres increased from $2.78 \times 10^{-4} \mathrm{~mol} \mathrm{~g}^{-1}$ with the MAA feeding amount of $100 \mu \mathrm{L}$ to $8.31 \times 10^{-4} \mathrm{~mol} \mathrm{~g}^{-1}$ with the MAA feeding amount of $700 \mu \mathrm{L}$ in the soap-free emulsion polymerization. The results show that the surface carboxyl group content of the microspheres increased with increasing the MAA feeding ratio. Fluorescent microspheres with different surface carboxyl group contents are prepared by the swellingevaporation method under optimal swelling conditions obtained in the previous research. The morphologies of the PSM microspheres with

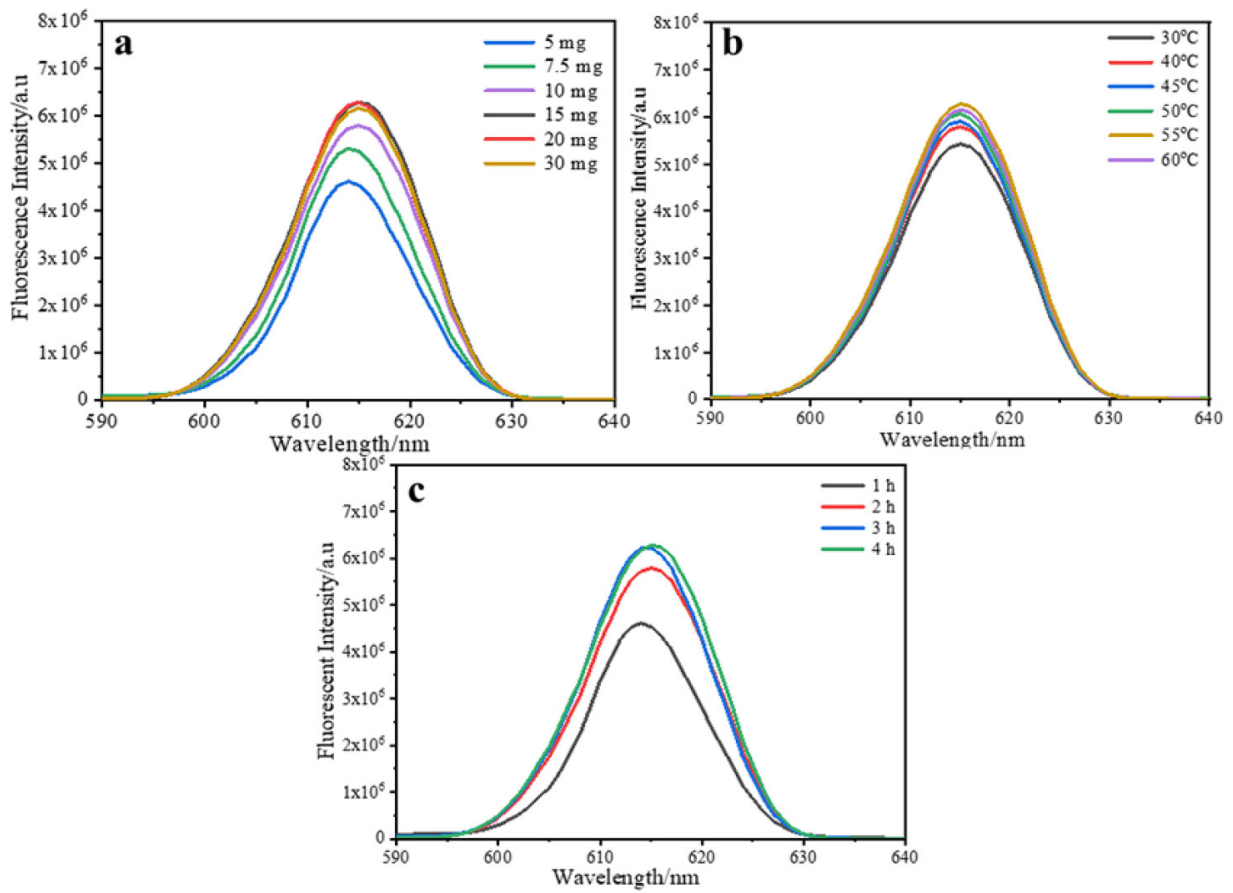

different surface carboxyl group contents are investigated by SEM (Figure 3S). It can be seen that almost all of the PSM microspheres (Figure 3Sa-3Se) are well dispersed with homogeneous spherical shapes and smooth surface, little change in particle size of microspheres. However, when the volume of MAA is further increased to 0.6-0.7 $\mathrm{mL}$, the sphericity of the products is reduced and ellipsoid is formed (Figure 3Sf, 3Sg). The contents of carboxyl group on the surface of the microspheres are $7.62 \times 10^{-4} \mathrm{~mol} \mathrm{~g}^{-1}$ and $8.31 \times 10^{-4} \mathrm{~mol} \mathrm{~g}^{-1}$. The results indicate that the concentration of MAA has an effect on the sphericity of the microspheres. If the volume of MAA is too much, the system will become unstable, resulting in failure to become spherical. It is confirmed from the TEM image of the luminescent composite microspheres containing $6.94 \times 10^{-4} \mathrm{~mol} \mathrm{~g}^{-1}$ carboxyl groups that the europium complex molecules are successfully incorporated into the interior of microspheres (Figure 3Sh).

Figure $5 \mathrm{~b}$ shows the fluorescence emission spectra of the fluorescent microspheres with different carboxyl group contents, the concentration of fluorescent microspheres is $3.3 \times 10^{-10} \mathrm{~mol} \mathrm{~L}^{-1}$. The fluorescence intensity of fluorescent microspheres first increases and then decreases with the increase of carboxyl group content, and as the carboxyl group content of the surface of microspheres reaches $6.94 \times 10^{-4} \mathrm{~mol} \mathrm{~g}^{-1}$, the fluorescence intensity reaches the maximum value. The reason of this result is because the carboxyl groups which bury within the microsphere also have a positive influence on the enhancement of fluorescence intensity. Because of the charge attraction between europium complex molecules and carboxyl groups, the europium complex on the surface of microspheres can easily enter the interior of microsphere. But the carboxyl contents are further increased, the steric hindrance the surface is increased, which effects the migration of europium complex to the interior of the microspheres, so the fluorescence intensity is decreased.

\section{Effect of the molecular weight of microspheres on the swelling properties of microspheres}

Considering that the concentration of initiator can affect the molecular weight of microspheres, when the concentration of 

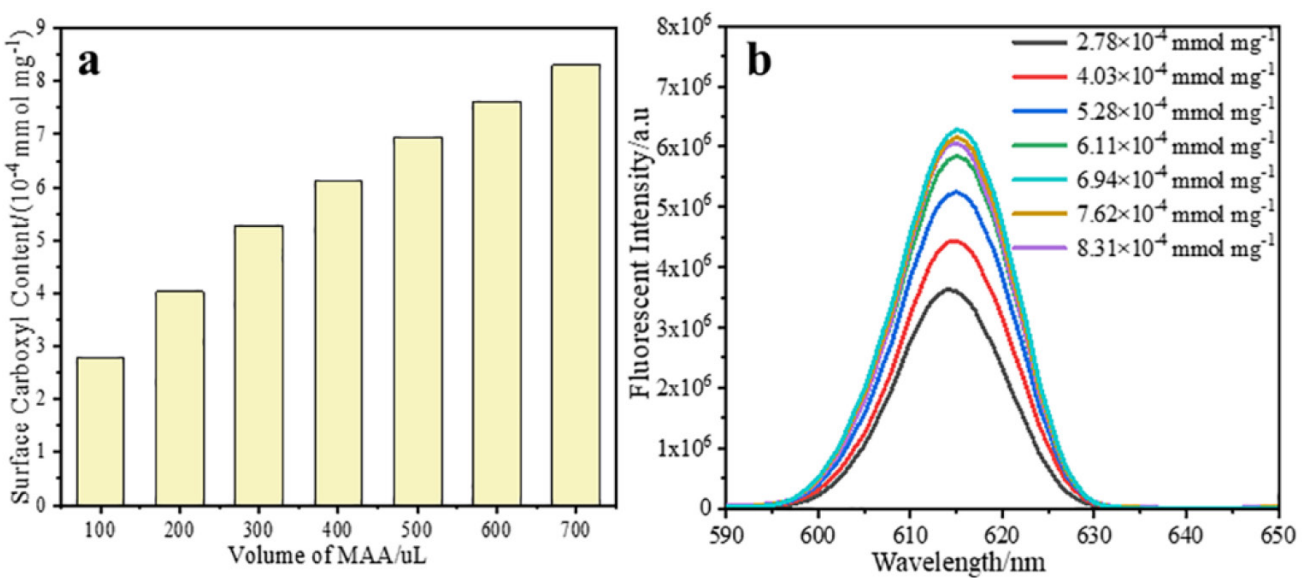

Figure 5. (a) The surface carboxyl content with increasing the volume of MAA; (b) Fluorescence emission spectra of fluorescent microspheres (the europium complex is $\left.8.7 \times 10^{-3} \mathrm{~mol} \mathrm{~L}^{-1}\right)$ with different carboxyl group contents

initiator increases, the rate of free radical formation is accelerated, the concentration of free radical in the continuous phase increases, the chain termination rate reaches, and the molecular weight of the polymer microspheres decreases. In the paper, keeping the volume of MAA all are $0.5 \mathrm{~mL}$, six types of polymer microspheres with different molecular weight are prepared with soap-free emulsion polymerization by adjusting the concentration of initiator. The molecular weight of microspheres is measured by gel permeation chromatography (GPC). The effect of initiator concentration on the molecular weight is shown in Figure 6a. It is apparent that the molecular weight decreases with increasing initiator concentration. The fluorescent microspheres are prepared by swelling the microsphere with different molecular weight.

The fluorescence emission spectra of the fluorescent composite microspheres with different molecule weight are collected through the fluorescence spectrophotometer. The concentration of fluorescent microspheres is $3.3 \times 10^{-10} \mathrm{~mol} \mathrm{~L}^{-1}$, the molar concentration of the
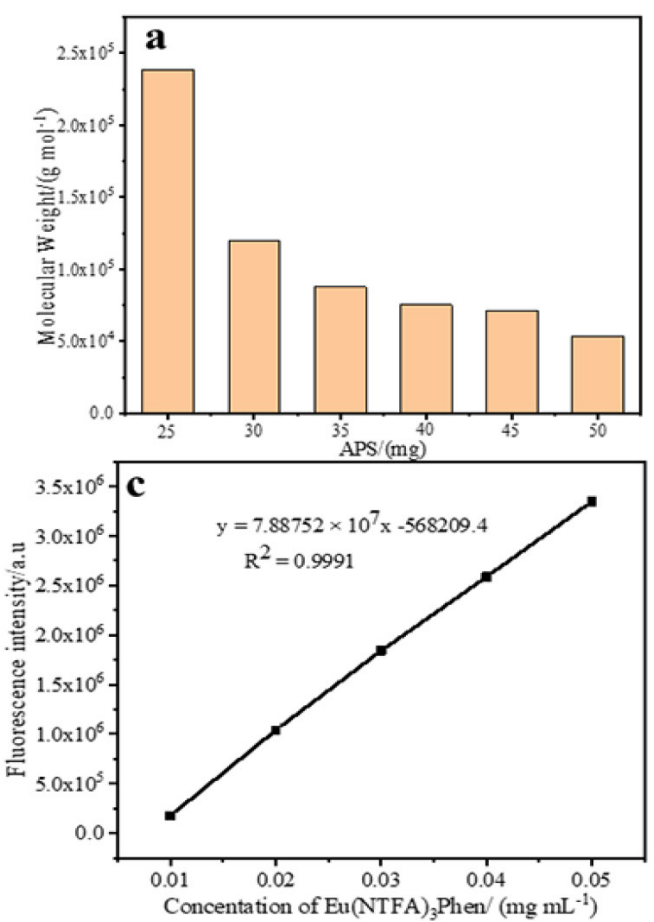

carboxyl groups on the surface of the microspheres is $6.94 \times 10^{-4} \mathrm{~mol} \mathrm{~g}^{-1}$. As illustrated in the Figure 6b, the fluorescence intensity of fluorescent microspheres first increases and then decreases with the decrease of molecular weight, and when the molecular weight of the microsphere is $75276 \mathrm{~g} \mathrm{~mol}^{-1}$, the fluorescence intensity reaches the maximum value. This is because when the molecular weight of microspheres is large, the structure of microspheres is tight and microspheres not easy to swell, so the europium complex molecules are difficult to migrate into microspheres. When the molecular weight of microspheres is small, the structure of microspheres is loose and easy for swelling which is conducive to the majority of fluorescent molecules incorporated into the inside of microspheres. But when the molecular weight of microspheres further reduction, microspheres structure is unstable, the europium complex molecules are easily spilled from the microspheres, so the fluorescence intensity is decreased.

In addition, the standard curve method is used to determine the mass of europium complex swelled into the microspheres.
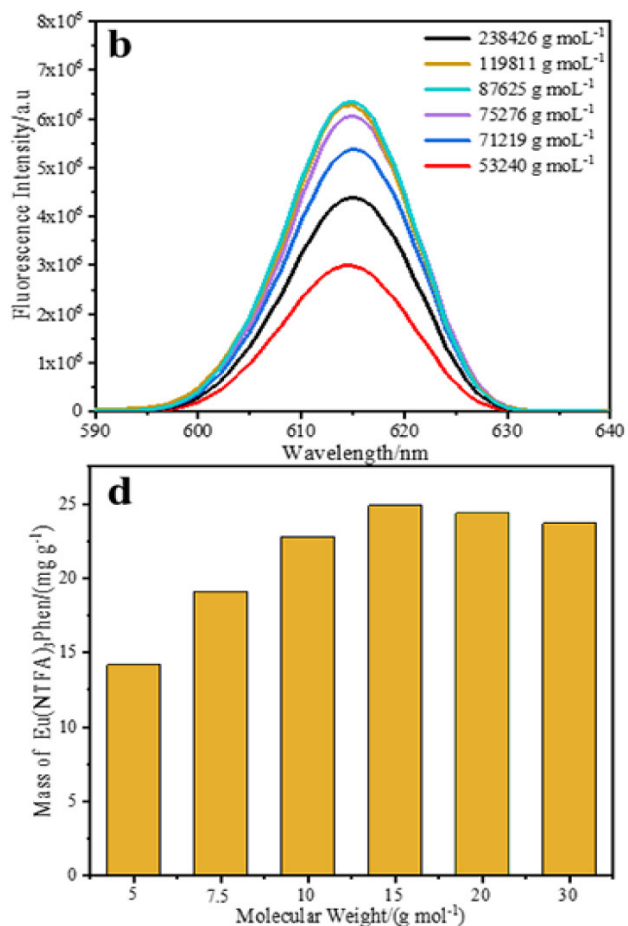

Figure 6. (a) The molecular weight of microspheres is changed with concentration of APS; (b) Fluorescence emission spectra of fluorescent microspheres with different molecular weight; (c) Standard curve of fluorescence intensity change based on the europium concentration in THF; (d) The mass of Eu(NTFA) ${ }_{3} P$ hen swelled into microspheres are changed with molecular weight 

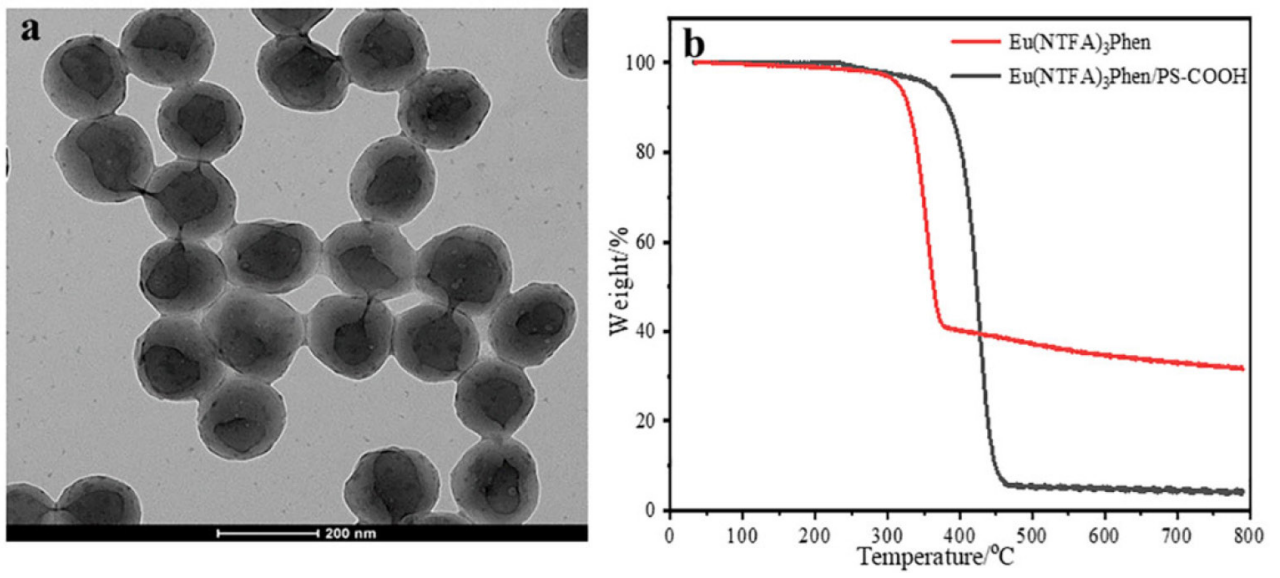

Figure 7. (a) TEM photomicrographs of fluorescent microspheres with molecular weight of $75276 \mathrm{~g} \mathrm{~mol}^{-1}$; (b) TGA thermograms of Eu(NTFA) ${ }_{3}$ Phen and $\mathrm{Eu}(\mathrm{NTFA})_{3}$ Phen/PS-COOH microspheres

The fluorescent microspheres with different molecular weight are dissolved in THF and released the europium complex molecules. Then europium complex dissolves in THF and measure the fluorescent intensity. The obtained fluorescent intensity is compared with the standard curve (Figure 6c) and calculated the mass of europium complex swelled into the polymer microsphere. As shown in Figure 6d, the mass of europium complex entering the microspheres reaches the maximum with a value of $24.9 \mathrm{mg} \mathrm{g}^{-1}$ when the surface carboxyl group content is $6.94 \times 10^{-4} \mathrm{~mol} \mathrm{~g}^{-1}$ and molecular weight is $75276 \mathrm{~g} \mathrm{~mol}^{-1}$, which is consistent with the previous results. Compared to the europium contents reported before, ${ }^{37}$ the europium content in the microspheres is increased about three times and the fluorescence intensity is greatly enhanced in this article.

The morphologies of fluorescent microspheres with different molecular weight are investigated by SEM (Figure 4S). It can be observed that all the microspheres own uniform diameter, homogeneous spherical shapes and smooth surface (Figure 4Sa-4Sd). However, with the molecular weight of microspheres increasing, the mean degree of diameter of the fluorescent microspheres decreases slightly (Figure 4Se, 4Sf). It is confirmed from the TEM image of the luminescent composite microspheres (Molecular weight: $75276 \mathrm{~g} \mathrm{~mol}^{-1}$ ) that the europium complex molecules are successfully incorporated into the interior of microspheres (Figure 7a).

To determine the thermal stability of the Eu/PS-COOH microspheres, TGA experiment are performed, the results are shown in Figure 7b. The weight loss of Eu/PS-COOH ( 3\% wt) below $290{ }^{\circ} \mathrm{C}$ is attributed to the loss of the residual solvent. ${ }^{38}$ The decomposition temperatures of the europium complex and $\mathrm{Eu} / \mathrm{PS}-\mathrm{COOH}$ microspheres are about $320{ }^{\circ} \mathrm{C}$ and $385{ }^{\circ} \mathrm{C}$, respectively. It can be seen from Figure $7 \mathrm{~b}$ thermal stability of $\mathrm{Eu} / \mathrm{PS}-\mathrm{COOH}$ microspheres is better than the pure europium complex. The reason is that the rigid PS microspheres provide a protect environment for the pure complex to make the europium complex more stable.

\section{CONCLUSIONS}

The paper focuses on the influence of the molecular weight and surface carboxyl group content of the microspheres on the swelling properties. Firstly, seven kinds of microspheres with different surface carboxyl group contents are successfully prepared. Secondly, the six kinds of microspheres with different molecular weight are prepared on the basic of optimal volume of MAA. Finally, the dispersed polystyrene fluorescent microspheres contained europium complex are prepared by swelling-evaporation method under the optional swelling conditions. The molecular weight of the microspheres is $75276 \mathrm{~g} \mathrm{~mol}^{-1}$, the surface carboxyl content is $6.94 \times 10^{-4} \mathrm{~mol} \mathrm{~g}^{-1}$, and contained $24.9 \mathrm{mg} \mathrm{g}^{-1}$ europium complex. The results indicate that the fluorescent microspheres have excellent fluorescence properties and abundant functional groups, which has important applications in biomolecules detection.

\section{SUPPLEMENTARY MATERIAL}

Figures $1 \mathrm{~S}-4 \mathrm{~S}$ containing fluorescence spectra and SEM images of the studied materials are freely available at http://quimicanova. sbq.org.br, in pdf format.

\section{ACKNOWLEDGMENTS}

This work was supported by the Fundamental Research Funds for the Central Universities (3207045420); the National Science Foundation for Cultivation of Bengbu University (2018GJPY03); Starting Research Fund of Bengbu University (BBXY2018KYQD15); and the National Natural Science Foundation of China (81703366).

\section{REFERENCES}

1. Zhang, X.; Wang, K.; Liu, M.; Zhang, X.; Tao, L.; Chen, Y.; Wei, Y.; Nanoscale 2015, 7, 11486.

2. Jiang, R.; Liu, M.; Huang, H.; Mao, L.; Huang, Q.; Wen, Y.; Cao, Q.; Tian, J.; Zhang, X.; Wei, Y.; J. Colloid Interface Sci. 2018, 519, 137.

3. Generalova, A. N.; Sizova, S. V.; Zdobnova, T. A.; Zarifullina, M. M.; Artemyev, M. V.; Baranov, A. V.; Oleinikov, V. A.; Zubov, P. V.; Deyev, S. M.; Nanomedicine 2011, 6, 196.

4. Wu, J.; Ye, Z. Q.; Wang, G. L.; Jin, D. Y.; Yuan, J. L.; Guan, Y. F.; James, P.; J. Mater. Chem. 2009, 19, 1259.

5. Sourov, C.; Pradip, D.; Dipranjan, L.; Sourav, B.; Panchanan, P.; Nanoscale 2011, 3, 1533.

6. Marta, B.; Raghuram, D.; Janos, V.; Biosens. Bioelectron. 2009, 24 , 1195 .

7. Shen, Q. X.; Xu, L. T.; Jiang, Y. Y.; Zhang, Y. P.; Pure. Appl. Chem. 2021, 3, 2231.

8. Parlak, O.; Demir, M. M.; ACS. Appl. Mater. Inter. 2011, 3, 4306.

9. Zhao, J.; Liu, Y. L.; Cheng, J. L.; Wu, S. M.; Wang, Z. B.; Hu, H. Q.; Zhou, C. H.; Polym. Int. 2017, 66, 1827.

10. Gu, H. B.; Ma, C.; Liang, C. B.; Meng, X. D.; Gu, J. W.; Guo, Z. H.; J. Mater. Chem C 2017, 5, 4275.

11. Anitha, S.; Asli, C.; Sumeyra, B.; Mesut, G.; Erdinc, D.; Faruk, Y.; Tamer, U.; ACS. Appl. Mater. Inter. 2015, 7, 21038. 
12. Shunmugam, R.; Tew, G. N.; Macromol. Rapid. Commun. 2008, 29, 1355.

13. Salas-Juarez, C. J.; Navarro, R. E.; Perez-Rodriguze, A.; OrozcoValencia, R.; Sensors Actuators, A 2020, 315, 0924

14. Xu, Z. H.; Bi, Y. F.; Yu, He.; Lin, J. Y.; Ding, F.; Sun, Y. G.; Gao, Y.; New J. Chem. 2017, 17, 1144.

15. Zhang, X. P.; Wen, S. P.; Hu, S.; Zhang, L. Q.; Liu, L.; J. Rare Earth 2010, 28, 333 .

16. Wang, H. G.; Yang, Q. B.; Sun, L.; Zhang, C. Q.; Li, Y. C.; Wang, S.; Li, Y. X.; J. Alloy Compd. 2009, 488, 414.

17. Princy, P.; Tomlal, J.; Divya, K. V.; Kuthanappilly, J. M.; Polym.-Plast Technol. Mater. 2021, 60, 886.

18. Manzani, D.; Nigoghossian, K.; Iastrensk, M. F.; Coelho, G. R.; Santos, M. V.; Maia, L. J. Q.; Ribeiro, S. J. L.; Segatelli, M. G.; J. Mater. Chem. C 2018, 30, 2050.

19. Summer, J. P.; Kopelman, R.; Analyst 2005, 130, 528.

20. Summer, J. P.; Westerberg, N. M.; Sroddard, A. K.; Fierke, C. A.; Kopelman, R.; Sensors Actuators, B 2006, 113, 760.

21. Ando, K.; Kawaguchi, H.; J. Colloid Interface Sci. 2005, 285, 610.

22. Sun, H. H.; Scharff-Poulsen, A. M.; Gu, H.; Kristoffer, A.; Mater. Chem. Phys. 2006, 18, 3381.

23. Xu, D. Z.; Liu, M. Y.; Huang, Q.; Chen, J. Y.; Huang, H. Y.; Deng, F. J.; Wen, Y. Q.; Tian, J. W.; Zhang, X. Y.; Wei, Y.; Talanta 2018, 188, 1.

24. Desbiens, J.; Bergeron, B.; Patry, M.; Ritcey, A. M.; J. Colloid Interface Sci. 2012, 376, 12

25. Li, P.; Song, Y.; Liu, C.; Li, X.; Zhou, G.; Fan, Y.; Mater. Lett. 2014, 114, 132 .
26. Behnke, T.; Wurth, C.; Laux, E. M.; Hoffmann, K.; Ute, R. G.; Dyes Pigm. 2012, 94, 247.

27. Frigoli, M.; Ouadahi, K.; Larpent, C.; Chem. - Eur. J. 2009, 15, 8319.

28. Fonseca, T.; Relogio, P.; Martinho, J. M. G.; Farinha, J. P. S.; Langmuir 2007, 23, 5727 .

29. Wang, L.; Lofton, C.; Popp, M.; Tan, W. H.; Bioconjugate Chem. 2007, 18,610 .

30. Pan, X. H.; Ju, J. H.; Li, J. J.; Wu, D. C.; High Perform. Polym. 2011, $23,255$.

31. Macrino, C. J.; Silva, E. M.; Cunha. V. R.; Fonseca, V. R.; Cunha Neto, Á.; Araujo, J. R.; Lacerda Jr., V.; Romão, W.; J. Braz. Chem. Soc. 2021, 32, 1070.

32. Xu, J. S.; Chen, D. L.; Hu, X. L.; Ke, Y. C.; Zhou, Q.; Gao, W. S.; Zeng, Z. L.; Zhang, G. L.; J. Polym. Eng. 2015, 35, 847.

33. Yuan, H. N.; He, G. G.; Compos. Interface 2020, published ahead of print; https://doi.org/ 10.1080/09276440.2020.1812948.

34. Wang, W.; Deng, Y.; Zhang, L. M.; Fu, J.; Lu, Z. X.; Xu, L. J.; J. Nanosci. Nanotechnol. 2012, 12, 7206.

35. Janczewski, D.; Song, J.; Julius-Vancso, G.; Eur. Polym. J. 2014, 54, 87.

36. Wang, S. Q.; Zhang, H. P.; Chen, X.; Li, H.; Wang, H.; Li, P. T.; Zhang, B. L.; Zhang, Q. Y.; RSC Adv. 2015, 5, 33554.

37. Wang, L. J.; Wang, X.; Wang, T. G.; Hu, Z. J.; Zou, G.; Zhang, Q. J.; J. Mater. Sci. 2012, 47, 2600.

38. Du, H. L.; Zhang, W.; Li, M.; Mao, M. F.; Luan, F. F.; Li, G. Z.; Peng, S. Q.; Kuang, Y. Q.; Guo, D. C.; J. Photochem. Photobiol., A 2020, 389, 112221 . 